\title{
COMMUTATORS IN REAL INTERPOLATION WITH QUASI-POWER PARAMETERS
}

\author{
MING FAN
}

Received 20 December 2001

The basic higher order commutator theorem is formulated for the real interpolation methods associated with the quasi-power parameters, that is, the function spaces on which Hardy inequalities are valid. This theorem unifies and extends various results given by Cwikel, Jawerth, Milman, Rochberg, and others, and incorporates some results of Kalton to the context of commutator estimates for the real interpolation methods.

\section{Introduction}

A current topic in interpolation theory is to estimate the nonlinear commutators in different situations. In the eighties, Rochberg, Weiss, and Jawerth initiated the study of the second order and abstract commutator theorems for scales of the classical complex and real interpolation spaces. The applications in many important differential and integral expressions were found ever since (see $[6,7,10,17]$ for details). The higher order commutator theorem was proved by Rochberg for the complex interpolation [16], and by Milman for the real interpolation [14]. Recently, these results were extended to the real interpolation with the Calderón weights [1]. In addition, Milman and Rochberg presented a comparison between the commutator results of the real and complex interpolation methods, and emphasized the role of internal cancellation in these results [15].

In the present paper, we formulate the basic higher order commutator theorem for a wide family of the real interpolation methods, the $K$ - and $J$-methods due to Brudnyı and Krugljak associated with the quasi-power parameters. This is motivated, in a large part, by the observation that the Hardy inequalities for the $L^{p}$-spaces $(1<p<\infty)$ play an important role in the proof of the commutator theorem for the classical interpolation methods. Furthermore, the 
quasilogarithmic operators in our commutator theorem is more general than the original ones. These operators, which are inspired by Kalton's work $[12,13]$, are determined by a much wide choice of Lipschitz functions. It therefore seems natural to us to carry over the commutator theorem to the real interpolation methods associated with the function spaces on which Hardy inequalities are valid. This unifies and extends various results given by Cwikel, Jawerth, Milman, Rochberg, and others, and incorporates some results of Kalton to the general theory of commutator estimates for the real interpolation methods.

This paper is organized as follows: in Section 2, we review the real interpolation methods with quasi-power parameters. In Section 3, we give definitions and results about quasilogarithmic operators corresponding to the above mentioned real interpolation methods. In Section 4, the main results about cancellation and commutators are formulated. Some useful remarks are included in Section 5.

Conventions. The notations $\subseteq$ and $=$ between Banach spaces stand for continuous inclusion and isomorphic equivalence, respectively. The notation $\mathscr{B}(X, Y)$, respectively, $\mathscr{B}(\bar{X}, \bar{Y})$, stands for the Banach space of all bounded linear operators from Banach space $X$ to Banach space $Y$, respectively, from Banach couple $\bar{X}$ to Banach couple $\bar{Y}$. We simply write $\mathscr{B}(X)=\mathscr{B}(X, X)$ and $\mathscr{B}(\bar{X})=\mathscr{B}(\bar{X}, \bar{X})$.

\section{Real interpolation with quasi-power parameters}

We suppose that the reader is familiar with the basic notations and definitions of interpolation theory, and we refer to $[3,4]$ for background information. We start by giving a brief review of the real interpolation methods with the quasi-power parameters.

Assume that $\bar{X}=\left(X_{0}, X_{1}\right)$ is a Banach couple with $\Delta \bar{X}=X_{0} \cap X_{1}$ and $\Sigma \bar{X}=$ $X_{0}+X_{1}$. For $t>0$, recall that the $J$ - and $K$-functionals on $\Delta \bar{X}$ and $\Sigma \bar{X}$, respectively, are given by

$$
\begin{aligned}
& J(t, x ; \bar{X})=\|x\|_{0} \vee\left(t\|x\|_{1}\right) \quad \text { if } x \in \Delta \bar{X}, \\
& K(t, x ; \bar{X})=\inf \left\{\left\|x_{0}\right\|_{0}+t\left\|x_{1}\right\|_{1} \mid x=x_{0}+x_{1}, x_{j} \in X_{j}\right\} \quad \text { if } x \in \Sigma \bar{X} .
\end{aligned}
$$

In particular, $J(1, x ; \bar{X})=\|x\|_{\Delta \bar{X}}$ and $K(1, x ; \bar{X})=\|x\|_{\Sigma \bar{X}}$. For simplicity, we usually write $J(t, x)=J(t, x ; \bar{X})$ and $K(t, x)=K(t, x ; \bar{X})$ when it does not lead to ambiguity. We now introduce Brudnyı̆-Krugljak's $K$ - and $J$-methods as follows.

Definition 2.1. Let $\Phi$ be a Banach function space over $\left(\mathbb{R}^{+}, d t / t\right)$ such that $1 \wedge t \in$ $\Phi$ and $\int_{0}^{\infty} 1 \wedge(1 / t)|f(t)| d t / t<\infty$ for all $f \in \Phi$. We define

$$
K_{\Phi}(\bar{X}):=\left\{x \in \Sigma \bar{X} \mid\|x\|_{K_{\Phi}}=\|K(t, x)\|_{\Phi}<\infty\right\} \quad[4,(3.3 .1)]
$$

and define $J_{\Phi}(\bar{X})$ as the space of all $x \in \Sigma \bar{X}$, which permits a canonical representation $x=\int_{0}^{\infty} u(t) d t / t$ for a strongly measurable function $u: \mathbb{R}^{+} \rightarrow \Delta \bar{X}$, with 
the norm

$$
\|x\|_{J_{\Phi}}=\inf _{u}\|J(t, u(t))\|_{\Phi}<\infty \quad[4,(3.4 .3)]
$$

Let $(\Omega, \mu)$ be a completely $\sigma$-finite measurable space and let $w$ be a weight on $(\Omega, \mu)$. We denote by $L^{0}(\Omega, d \mu)$ the space of all measurable functions on $(\Omega, \mu)$ with $\|f\|_{L^{0}}=\mu(\operatorname{supp} f)<\infty$, and denote by

$$
L^{p}(w d \mu)=\left\{f \mid\|f\|_{L^{p}(w d \mu)}=\left(\int_{\Omega}|f|^{p} w d \mu\right)^{1 / p}<\infty\right\},
$$

the weighted $L^{p}$-space for $1 \leq p<\infty$, with the usual change for $p=\infty$. For the measurable function $\varphi: \mathbb{R}^{+} \rightarrow \mathbb{R}^{+}$, we write

$$
L_{\varphi}^{p}=\left\{f \mid \frac{f}{\varphi} \in L^{p}\left(\mathbb{R}^{+}, \frac{d t}{t}\right)\right\}
$$

In particular, we write $L_{\theta}^{p}=L_{\varphi_{\theta}}^{p}$ for the power function $\varphi_{\theta}(t)=t^{\theta}$. Now let us define the Hardy operators $P, Q$ and the Calderón operator $S$ on $L^{0}\left(\mathbb{R}^{+}, d t / t\right)$ by

$$
\begin{gathered}
(P f)(t)=\int_{0}^{t} f(s) \frac{d s}{s}, \\
(Q f)(t)=t \int_{t}^{\infty} f(s) \frac{d s}{s^{2}} \\
(S f)(t)=(P f+Q f)(t)=\int_{0}^{\infty} 1 \wedge\left(\frac{t}{s}\right) f(s) \frac{d s}{s} .
\end{gathered}
$$

The Banach function space $\Phi$ is said to be a quasi-power parameter for the real interpolation if $S \in \mathscr{B}(\Phi)$. Observe that $S \in \mathscr{B}(\Phi)$ if and only if $P, Q \in \mathscr{B}(\Phi)$. In this case, we have the equivalence $J_{\Phi}(\bar{X})=K_{\Phi}(\bar{X})$ with the norm estimate

$$
\|x\|_{K_{\Phi}(\bar{X})} \leq\|S\|_{\mathscr{B}(\Phi)}\|x\|_{J_{\Phi}(\bar{X})}
$$

for all Banach couples $\bar{X}$ [4, Corollary 3.5.35]. A function $\varphi: \mathbb{R}^{+} \rightarrow \mathbb{R}^{+}$is said to be quasi-power if $S \varphi$ is equivalent to $\varphi$. The function space $L_{\varphi}^{p}$ is a quasi-power parameter for the real interpolation if $\varphi$ is a quasi-power function. We simply write $J_{\varphi}^{p}(\bar{X})=J_{L_{\varphi}^{p}}(\bar{X})$ and $K_{\varphi}^{p}(\bar{X})=K_{L_{\varphi}^{p}}(\bar{X})$.

The following examples show that several interpolation methods in the literature can be formulated in terms of Brudnyı-Krugljak's $K$ - and $J$-methods with the quasi-power parameters for the real interpolation. 
Example 2.2 (real methods associated with Calderón weights [1]). A weight $w$ on $\left(\mathbb{R}^{+}, d t\right)$ is said to be a Calderón weight if the Calderón type operator $\tilde{S} \in$ $\mathscr{B}\left(L^{p}(w d t)\right)$, where

$$
(\tilde{S} f)(t)=\int_{0}^{\infty}\left(\frac{1}{t} \wedge \frac{1}{s}\right) f(s) d s
$$

For a Banach couple $\bar{X}$, we define $\bar{X}_{p, w, K}$ to be the space of all those $x \in \Sigma \bar{X}$, for which $K(t, x) / t \in L^{p}(w d t)$, with the norm $\|x\|_{\bar{X}_{p, w, K}}=\|K(t, x) / t\|_{L^{p}(w d t)}$, and define $\bar{X}_{p, w, J}$ to be the space of all those $x \in \Sigma \bar{X}$, for which there exists a strongly measurable function $u: \mathbb{R}^{+} \rightarrow \Delta \bar{X}$ satisfying $x=\int_{0}^{\infty} u(t) d t / t$ and $J(t, u(t)) / t \in$ $L^{p}(w d t)$, with the norm $\|x\|_{\bar{X}_{p, w, J}}=\inf _{u}\left\{\|J(t, u(t)) / t\|_{L^{p}(w d t)}\right\}$. If we set $\varphi(t)=$ $t^{1-1 / p} w(t)^{-1 / p}$, then the space $L_{\varphi}^{p}$ is a quasi-power parameter for the real interpolation. By substituting $\Phi$ with $L_{\varphi}^{p}$ in (2.7), we obtain that

$$
\bar{X}_{p, w, J}=J_{\varphi}^{p}(\bar{X})=K_{\varphi}^{p}(\bar{X})=\bar{X}_{p, w, K} .
$$

Example 2.3 (E- and $E_{\alpha}$-methods $[4,10]$ ). Let $r>0$ and let $x \in \Sigma \bar{X}$. We first consider the $E$-functional which is given by

$$
E(r, x)=\inf \left\{\left\|x_{0}\right\|_{0} \mid x=x_{0}+x_{1}, x_{j} \in X_{j},\left\|x_{1}\right\|_{1} \leq r\right\} .
$$

For a quasi-power parameter $\Phi$ for the real interpolation, we set

$$
\Psi=K_{\Phi}\left(L^{\infty}\left(\frac{d r}{r}\right), L^{0}\left(\frac{d r}{r}\right)\right),
$$

and define the space $E_{\Psi}(\bar{X})$ consisting of all $x \in \Sigma \bar{X}$ such that

$$
\|x\|_{E_{\Psi}(\bar{X})}=\inf \left\{\lambda>0 \mid\left\|\lambda^{-1} E(\lambda r, x)\right\|_{\Psi} \leq 1\right\}<\infty .
$$

It is known that $E_{\Psi}(\bar{X})=K_{\Phi}(\bar{X})$ by [4, Theorem 4.2.25 and (4.2.38)]. Next, consider the $E_{\alpha}$-functional, $\alpha \geq 1$, which is given by

$$
\begin{aligned}
& E_{1}(r, x)=\inf \left\{\frac{\left\|x_{0}\right\|_{0}}{r} \mid x=x_{0}+x_{1}, x_{j} \in X_{j},\left\|x_{1}\right\|_{1} \leq r\right\}, \\
& E_{\alpha}(r, x)=\inf \left\{\left(\frac{\left\|x_{0}\right\|_{0}}{r}\right)^{1 / \alpha} \vee\left(\frac{\left\|x_{1}\right\|_{1}}{r}\right)^{1 /(\alpha-1)} \mid x=x_{0}+x_{1}, x_{j} \in X_{j}\right\}
\end{aligned}
$$

for $\alpha>1$. It is clear that $E(r, x)=r E_{1}(r, x)$. According to [10, Lemma 2.1], for each $x \in \Sigma \bar{X}$, the right continuous inverse of the function $E_{\alpha}(r, x)$ is $K(t, x) / t^{\alpha}$. For $p \geq 1$ and $0<\theta<1$, we choose $\eta=1 /(\theta-\alpha), q=p(\alpha-\theta)$ and define the space $\bar{X}_{\theta, p, E_{\alpha}}$ consisting of all $x \in \Sigma \bar{X}$ for which

$$
\|x\|_{\bar{X}_{\theta, p, E_{\alpha}}}=\left\|E_{\alpha}(r, x)\right\|_{L_{\eta}^{q}}^{\alpha-\theta}<\infty .
$$


By the change of variable $r=K(t, x) / t^{\alpha}$ and integrating by parts, we have

$$
\bar{X}_{\theta, p, E_{\alpha}}=K_{\theta}^{p}(\bar{X}) .
$$

\section{Quasilogarithmic operators}

Now consider the relevant definitions and results about quasilogarithmic operators in the real interpolation, which came originally from [10]. In this section, however, those operators will be considered in a more general sense. The main result here is to present the connection between the quasilogarithmic operators in different versions of the real interpolation methods. In this and the next section, we always assume that $\Phi$ is a quasi-power parameter for the real interpolation.

Let $\bar{X}$ be a Banach couple, and let $c>1$ be a constant which is fixed during the discussion in this section. For $x \in \Sigma \bar{X}$, the decomposition $x=x_{0}(t)+x_{1}(t), t>0$, is (c-)almost optimal for the $K$-methods if

$$
K(t, x) \leq\left\|x_{0}(t)\right\|_{0}+t\left\|x_{1}(t)\right\|_{1} \leq c K(t, x) .
$$

A $(c$-)almost optimal projection for the $K$-methods is a (usually nonlinear) operator $D_{K}(t): \Sigma \bar{X} \rightarrow X_{0}$ defined by

$$
D_{K}(t) x=D_{K}(t, \bar{X}) x=x_{0}(t)
$$

for some almost optimal decomposition. It is always possible to choose $x_{j}(t)$ $(j=0,1)$ to be continuous. Now we consider the $J_{\Phi}$ methods. Given $x \in \Sigma \bar{X}$, we say that

$$
D_{J}(t) x=D_{J, \Phi}(t, \bar{X}) x=u(t)
$$

defines a (c-)almost optimal decomposition for the $J_{\Phi}$ method if $u: \mathbb{R}^{+} \rightarrow \Delta \bar{X}$ satisfies $x=\int_{0}^{\infty} u(t) d t / t$ in $\Sigma \bar{X}$ and $\|J(t, u(t))\|_{\Phi} \leq c\|x\|_{J_{\Phi}}$ for $t>0$.

Here and throughout, we assume that $\psi: \mathbb{R} \rightarrow \mathbb{R}$ is a Lipschitz function satisfying

$$
|\psi(t)-\psi(s)| \leq \gamma_{\psi}|t-s|
$$

where $\gamma_{\psi}$ is a constant depending on $\psi$. For $n=1,2, \ldots$, we introduce the following concept.

Definition 3.1. The quasilogarithmic operators $\Omega_{\psi, n}^{J}$ and $\Omega_{\psi, n}^{K}$ of order $n$ are defined on $\Sigma \bar{X}$ in the following way

$$
\begin{aligned}
& \Omega_{\psi, n}^{J}(x)=\frac{1}{n !} \int_{0}^{\infty} \psi(\log t)^{n} D_{J}(t) x \frac{d t}{t}, \\
& \Omega_{\psi, n}^{K}(x)=\frac{1}{n !} \int_{0}^{\infty}\left(I \cdot \chi_{(1, \infty)}(t)-D_{K}(t)\right) x d \psi(\log t)^{n}+\frac{1}{n !} \psi(0)^{n} x .
\end{aligned}
$$


In particular, if $\psi(t)=t$, then $\Omega_{\psi, n}^{J}=\Omega_{n, J}$ and $\Omega_{\psi, n}^{K}=-\Omega_{n, K}$ as given in [15].

Remark 3.2. For the $E_{\alpha}$-methods, respectively, $E$-methods, we can also introduce the almost optimal decomposition $x=x_{0}(r)+x_{1}(r), r>0$, and the almost optimal projection $D_{E_{\alpha}}(r) x=x_{0}(r)$, respectively, $D_{E}(r) x=x_{0}(r)$, where

$$
E_{\alpha}(r, x) \leq\left(\frac{\left\|x_{0}(r)\right\|_{0}}{r}\right)^{1 / \alpha} \vee\left(\frac{\left\|x_{1}(r)\right\|_{1}}{r}\right)^{1 /(\alpha-1)} \leq E_{\alpha}\left(\frac{r}{c}, x\right)
$$

for $\alpha>1$, and

$$
E_{1}(r, x) \leq \frac{\left\|x_{0}(r)\right\|_{0}}{r} \leq E_{1}\left(\frac{r}{c}, x\right), \quad\left\|x_{1}(r)\right\|_{1} \leq r,
$$

respectively,

$$
E(r, x) \leq\left\|x_{0}(r)\right\|_{0} \leq E\left(\frac{r}{c}, x\right), \quad\left\|x_{1}(r)\right\|_{1} \leq r .
$$

The corresponding quasilogarithmic operator $\Omega_{\psi, n}^{\alpha}=\Omega_{\psi, n}^{E_{\alpha}}$, respectively, $\Omega_{\psi, n}^{E}$, can be similarly defined by

$$
\begin{aligned}
& \Omega_{\psi, n}^{\alpha}(x)=\frac{1}{n !} \int_{0}^{\infty}\left(I \cdot \chi_{(1, \infty)}(r)-D_{E_{\alpha}}(r)\right) x d \psi(\log r)^{n}+\frac{1}{n !} \psi\left(\log \|x\|_{\Sigma \bar{X}}\right)^{n} x, \\
& \Omega_{\psi, n}^{E}(x)=\frac{1}{n !} \int_{0}^{\infty}\left(I \cdot \chi_{(1, \infty)}(r)-D_{E}(r)\right) x d \psi(\log r)^{n}+\frac{1}{n !} \psi\left(\log \|x\|_{\Sigma \bar{X}}\right)^{n} x .
\end{aligned}
$$

Before proceeding, recall some basic facts about rearrangement invariant function spaces, which can be found in [2]. Let $(\Omega, \mu)$ be a completely $\sigma$-finite measurable space as before. For $f \in L^{0}(\Omega, d \mu)$, we denote the rank function of $f$ by

$$
r_{f}(\omega)=\mu(\{\eta \in \Omega|| f(\eta)|>| f(\omega) \mid\}) \quad \text { for } \omega \in \Omega,
$$

the distribution function of $f$ by

$$
\lambda_{f}(s)=\mu(\{\omega \in \Omega|| f(\omega) \mid>s\}) \quad \text { for } s>0
$$

and the measure-preserving rearrangement of $|f|$ by

$$
f^{*}(t)=\inf \left\{s \mid \lambda_{f}(s) \leq t\right\} \quad \text { for } t>0 .
$$

Two functions in $L^{0}(\Omega, d \mu)$ are said to be equimeasurable if they have the same distribution function. A Banach function space $X$ over $(\Omega, d \mu)$ is said to be rearrangement invariant (r.i. in short) if $\|f\|_{X}=\|g\|_{X}$ for every pair of equimeasurable functions $f$ and $g$ in $L^{0}(\Omega, d \mu)$. We refer to [2, Definitions III.5.10 and III.5.12] for the Boyd indices of r.i. spaces. 
Lemma 3.3. Assume that $\Phi$ is a Banach function space over $\left(\mathbb{R}^{+}, d t / t\right)$ such that the Calderón operator $S$ is bounded on $\Phi$. Let

$$
\Phi^{*}=\left\{f \in L^{0}\left(\mathbb{R}^{+}, d t\right) \mid t f^{*}(t) \in \Phi\right\}
$$

with the norm $\|f\|_{\Phi^{*}}=\left\|t f^{*}(t)\right\|_{\Phi}$. Then $\Phi^{*}$ is an r.i. space over $\left(\mathbb{R}^{+}, d t\right)$ with the Boyd indices $\alpha$, $\beta$ satisfying $0<\alpha \leq \beta<1$.

Proof. First we introduce the Banach function space $\tilde{\Phi}$ over $\left(\mathbb{R}^{+}, d t\right)$ by

$$
\tilde{\Phi}=\left\{f \in L^{0}\left(\mathbb{R}^{+}, d t\right) \mid t f(t) \in \Phi\right\}
$$

with the norm $\|f\|_{\tilde{\Phi}}=\|t f(t)\|_{\Phi}$. Observe that the operator $\tilde{S}$ given by (2.8) is bounded on $\tilde{\Phi}$ with $\|\tilde{S}\|_{\mathscr{B}(\tilde{\Phi})}=\|S\|_{\mathscr{B}(\Phi)}$. In fact, for $f \in L^{0}\left(\mathbb{R}^{+}, d t\right)$, we have

$$
\begin{aligned}
\|\tilde{S} f\|_{\tilde{\Phi}} & =\left\|t \int_{0}^{\infty}\left(\frac{1}{t} \wedge \frac{1}{s}\right) f(s) d s\right\|_{\Phi}=\left\|\int_{0}^{\infty}\left(1 \wedge \frac{t}{s}\right) s f(s) \frac{d s}{s}\right\|_{\Phi} \\
& \leq\|S\|_{\Re(\Phi)}\|s f(s)\|_{\Phi}=\|S\|_{\mathscr{B}(\Phi)}\|f\|_{\tilde{\Phi}},
\end{aligned}
$$

and similarly $\|S f\|_{\Phi} \leq\|\tilde{S}\|_{\mathscr{B}(\tilde{\Phi})}\|f\|_{\Phi}$. The space $\Phi^{*}$ is an r.i. space over $\left(\mathbb{R}^{+}, d t\right)$ due to the equalities

$$
\left\|f^{*}\right\|_{\Phi^{*}}=\left\|\left(f^{*}\right)^{*}\right\|_{\tilde{\Phi}}=\left\|f^{*}\right\|_{\tilde{\Phi}}=\|f\|_{\Phi^{*}}
$$

These observations, together with [2, III. (5.7)], imply that

$$
\|\tilde{S} f\|_{\Phi^{*}}=\left\|(\tilde{S} f)^{*}\right\|_{\tilde{\Phi}} \leq\left\|\tilde{S} f^{*}\right\|_{\tilde{\Phi}} \leq\|\tilde{S}\|_{\mathscr{B}(\tilde{\Phi})}\left\|f^{*}\right\|_{\tilde{\Phi}}=\|\tilde{S}\|_{\mathscr{B}(\tilde{\Phi})}\|f\|_{\Phi^{*}}
$$

That is, the operator $\tilde{S}$ is also bounded on $\Phi^{*}$. Following [2, Theorem III. 5.14], the Boyd indices $\alpha$ and $\beta$ of $\Phi^{*}$ satisfying $0<\alpha \leq \beta<1$.

The main tools to be used in the course of the proof for the main theorems in this and the next section are integrating by parts and internal cancellation. The following lemma is crucial for this reason. We refer to [5, Lemma 3.2] for the classical case.

LemMA 3.4. Let $\Phi$ be a quasi-power parameter for the real interpolation, let $\psi$ be a Lipschitz function satisfying (3.4), and let $u: \mathbb{R}^{+} \rightarrow \Delta \bar{X}$, for which $\int_{0}^{\infty} u(t) d t / t=0$ and $\|J(t, u(t))\|_{\Phi}<\infty$. Then, for $n \geq 1$,

$$
\psi(\log t)^{n} \int_{0}^{t} u(s) \frac{d s}{s} \longrightarrow 0 \quad \text { in } \Sigma \bar{X}
$$

as $t \rightarrow 0$ or $\infty$.

Proof. We begin with the special case $\psi(t)=t$. Let $\Phi^{*}$ and $\tilde{\Phi}$ be given as in (3.14) and (3.15). Then the Boyd indices $\alpha, \beta$ of $\Phi^{*}$ satisfy $0<\alpha \leq \beta<1$ by Lemma 3.3. 
If we choose $p_{0}$ and $p_{1}$ such that $1<p_{0}<1 / \beta \leq 1 / \alpha<p_{1}<\infty$, then, by the Boyd interpolation theorem [2, Theorem III.5.16], $\Phi^{*}$ is an interpolation space for Banach couple $\left(L^{p_{0}}, L^{p_{1}}\right)$, where $L^{p_{j}}=L^{p_{j}}\left(\mathbb{R}^{+}, d t\right)(j=0,1)$. Thus, $\Phi^{*} \subseteq L^{p_{0}}+L^{p_{1}}$. By [9, Theorem 4.1], there is a constant $C>0$,

$$
\left(\int_{0}^{1} f^{*}(s)^{p_{0}} d s\right)^{1 / p_{0}}+\left(\int_{1}^{\infty} f^{*}(s)^{p_{1}} d s\right)^{1 / p} \leq C\|f\|_{L^{p_{0}}+L^{p_{1}}}
$$

for all $f \in L^{p_{0}}+L^{p_{1}}$. For $x \in \Phi$, let $K(t)=K(t, x)$. Then $(K(t) / t)^{*}=K(t) / t$ since $K(t) / t$ is decreasing on $\mathbb{R}^{+}$by [3, Lemma 3.1.1]. Moreover,

$$
\|K(t)\|_{\Phi}=\left\|\frac{K(t)}{t}\right\|_{\tilde{\Phi}}=\left\|\left(\frac{K(t)}{t}\right)^{*}\right\|_{\tilde{\Phi}}=\left\|\frac{K(t)}{t}\right\|_{\Phi^{*}} .
$$

Now let $u$ be given by the assumption. For fixed $t>0$, let

$$
u_{t}(s)=u(s) \cdot \chi_{(0, t]}(s), \quad x_{t}=\int_{0}^{t} u(s) \frac{d s}{s}=\int_{0}^{\infty} u_{t}(s) \frac{d s}{s} .
$$

Then $J\left(s, u_{t}(s)\right) \leq J(s, u(s))$, and $J\left(s, u_{t}(s)\right) \leq 2 K\left(s, x_{t}\right)$ by the fundamental inequality for the real interpolation [11]. This implies that

$$
\left\|x_{t}\right\|_{\Sigma \bar{X}} \leq \int_{0}^{t}\|u(s)\|_{0} \frac{d s}{s} \leq \int_{0}^{t} J\left(s, u_{t}(s)\right) \frac{d s}{s} \leq 2 \int_{0}^{t} K\left(s, x_{t}\right) \frac{d s}{s} .
$$

Now we set $K_{t}(s)=K\left(s, x_{t}\right)$ and $\theta_{j}=1 / p_{j}^{\prime}(j=0,1)$, and assume that $t<1$. According to the Hölder inequality, (2.7), (3.20), and (3.21), we obtain

$$
\begin{aligned}
& \int_{0}^{t} K_{t}(s) \frac{d s}{s}=\int_{0}^{t} \frac{K_{t}(s)}{s^{\theta_{0}}} s^{\theta_{0}} \frac{d s}{s} \\
& \leq\left(\int_{0}^{t} s^{\theta_{0} p_{0}^{\prime}} \frac{d s}{s}\right)^{1 / p_{0}^{\prime}} \cdot\left(\int_{0}^{t}\left(\frac{K_{t}(s)}{s^{\theta_{0}}}\right)^{p_{0}} \frac{d s}{s}\right)^{1 / p_{0}} \\
& \leq t^{\theta_{0}}\left(\int_{0}^{1}\left(\frac{K_{t}(s)}{s}\right)^{p_{0}} d s\right)^{1 / p_{0}} \leq C t^{\theta_{0}}\left\|\frac{K_{t}(s)}{s}\right\|_{L^{p_{0}+L^{p_{1}}}} \\
& \leq C t^{\theta_{0}}\left\|\frac{K_{t}(s)}{s}\right\|_{\Phi^{*}}=C t^{\theta_{0}}\left\|K_{t}(s)\right\|_{\Phi} \\
& \leq C t^{\theta_{0}}\|S\|_{\mathscr{B}(\Phi)}\|J(s, u(s))\|_{\Phi} .
\end{aligned}
$$

Consequently, $(\log t)^{n} \int_{0}^{t} u(s) d s / s \rightarrow 0$ in $\Sigma \bar{X}$ as $t \rightarrow 0$ by a simple estimate. On the other hand, the cancellation assumption yields

$$
x_{t}=\int_{0}^{t} u(s) \frac{d s}{s}=-\int_{t}^{\infty} u(s) \frac{d s}{s} .
$$


For $t>1$, a similar estimate leads to

$$
\begin{aligned}
\left\|x_{t}\right\|_{\Sigma \bar{X}} & \leq \int_{t}^{\infty}\|u(s)\|_{1} \frac{d s}{s} \leq \int_{t}^{\infty} \frac{J(s, u(s))}{s} \frac{d s}{s} \leq 2 \int_{t}^{\infty} \frac{K_{t}(s)}{s} \frac{d s}{s} \\
& \leq 2\left(\int_{1}^{\infty} s^{\left(\theta_{1}-1\right) p_{1}^{\prime}} \frac{d s}{s}\right)^{1 / p_{1}^{\prime}} \cdot\left(\int_{t}^{\infty}\left(\frac{K_{t}(s)}{s^{\theta_{1}}}\right)^{p_{1}} \frac{d s}{s}\right)^{1 / p_{1}} \\
& \leq \frac{2 C}{t^{1-\theta_{1}}}\|S\|_{\mathscr{P}(\Phi)}\|J(s, u(s))\|_{\Phi} .
\end{aligned}
$$

Thus, the convergence $(\log t)^{n} \int_{0}^{t} u(s) d s / s \rightarrow 0$ holds in $\Sigma \bar{X}$ as $t \rightarrow \infty$.

Generally, for an arbitrary Lipschitz function $\psi$ satisfying (3.4), we have $|\psi(\log t)-\psi(0)| \leq \gamma_{\psi}|\log t|$, and

$$
\left|\psi(\log t)^{n}-\psi(0)^{n}\right|=|\psi(\log t)-\psi(0)| \cdot\left|\sum_{k=0}^{n-1} \psi(\log t)^{k} \psi(0)^{n-1-k}\right| .
$$

Therefore, we obtain

$$
\psi(\log t)^{n} \int_{0}^{t} u(s) \frac{d s}{s} \longrightarrow 0 \quad \text { in } \Sigma \bar{X} \text { as } t \longrightarrow 0 \text { or } \infty
$$

by a simple estimate for $n=1$ and by induction for $n>1$.

It is reasonable to extend [6, Theorem 2.8] as follows:

Theorem 3.5. Let $x \in \Sigma \bar{X}^{0}$, the closure of $\Delta \bar{X}$ in $\Sigma \bar{X}$. Then there is a decomposition $x=\int_{0}^{\infty} u(t) d t / t$ for the $J_{\Phi}$ methods, which is almost optimal simultaneously for all choices of quasi-power parameters $\Phi$ for the real interpolation. Furthermore, there exists an almost optimal projection of $x$ for the $K_{\Phi}$ methods such that

$$
\Omega_{\psi, n}^{J}(x)=\Omega_{\psi, n}^{K}(x)
$$

Proof. Let $x \in \Sigma \bar{X}^{0}$. As in the proof of [6, Theorem 2.8], we can find a decomposition

$$
x=\int_{0}^{\infty} u(s) \frac{d s}{s}=x_{0}(t)+x_{1}(t) \quad \text { for } t>0
$$

where $x_{0}(t)=\int_{0}^{t} u(s) d s / s \in X_{0}$ and $x_{1}(t)=\int_{1}^{\infty} u(s) d s / s \in X_{1}$ such that

$$
\left\|x_{0}(t)\right\|_{0}+t\left\|x_{1}(t)\right\|_{1} \leq c K(t, x) .
$$

That is, $D_{K}(t) x=x_{0}(t)$ is an almost optimal projection for the $K$-method. By the fundamental inequality for the real interpolation [11] and by the estimate in (2.7), we have

$$
\|J(t, u(t))\|_{\Phi} \leq 2\|K(t, x)\|_{\Phi}=2\|x\|_{K_{\Phi}} \leq 2\|S\|_{\mathscr{B}(\Phi)}\|x\|_{J_{\Phi}} .
$$


This implies that $D_{J}(t) x=u(t)$ is an almost optimal decomposition for the $J_{\Phi}$ methods for all choices of quasi-power parameters $\Phi$.

Further calculation gives that

$$
\begin{aligned}
\Omega_{\psi, n}^{K}(x)= & \frac{1}{n !} \int_{0}^{\infty}\left(I \cdot \chi_{(1, \infty)}(t)-D_{K}(t)\right) x d \psi(\log t)^{n}+\frac{1}{n !} \psi(0)^{n} x \\
= & \frac{1}{n !}\left(-\int_{0}^{1} \int_{0}^{t} u(s) \frac{d s}{s} d \psi(\log t)^{n}\right. \\
& \left.\quad+\int_{1}^{\infty} \int_{t}^{\infty} u(s) \frac{d s}{s} d \psi(\log t)^{n}+\psi(0)^{n} x\right) \\
= & \frac{1}{n !}\left(\int_{0}^{1} \psi(\log t)^{n} u(t) \frac{d t}{t}+\int_{1}^{\infty} \psi(\log t)^{n} u(t) \frac{d t}{t}\right) \\
= & \frac{1}{n !} \int_{0}^{\infty} \psi(\log t)^{n} D_{J}(t) x \frac{d t}{t}=\Omega_{\psi, n}^{K}(x) .
\end{aligned}
$$

Here we use Lemma 3.4 for integration by parts.

Remark 3.6. For the operator $\Omega_{\psi, n}^{\alpha}$ with $\alpha \geq 1$, as in Remark 3.2, we consider $\Phi=$ $L_{\theta}^{p}$. Let $K(t)=K(t, x)$ and $E_{\alpha}(r)=E_{\alpha}(t, x)$. If we use the change of variable $r=$ $K(t) / t^{\alpha}$, then $E_{\alpha}(r)=t$ for each $x \in \Sigma \bar{X}[10,(2.7)]$, and hence $D_{K}(t) x=D_{E_{\alpha}}(r) x$. Moreover, $1 \wedge t \leq K(t) \leq 1 \vee t$, and hence

$$
\alpha-1 \leq \alpha-\frac{\log K(t)}{\log t} \leq \alpha .
$$

This gives that

$$
\alpha-1 \leq\left|\frac{\log \left(K(t) / t^{\alpha}\right)}{\log t}\right| \leq \alpha .
$$

By using Lemma 3.4 again, we obtain

$$
\psi\left(\log \frac{K(t)}{t^{\alpha}}\right)^{n} \int_{0}^{t} u(s) \frac{d s}{s} \longrightarrow 0 \quad \text { as } t \longrightarrow 0 \text { or } \infty .
$$

It turns out

$$
\begin{aligned}
\Omega_{\psi, n}^{\alpha}(x) & =\frac{1}{n !} \int_{0}^{\infty}\left(I \cdot \chi_{(1, \infty)}(t)-D_{K}(t)\right) x d \psi\left(\log \frac{K(t)}{t^{\alpha}}\right)^{n}+\frac{1}{n !} \psi\left(\log \|x\|_{\Sigma \bar{X}}\right)^{n} x \\
& =\frac{1}{n !} \int_{0}^{\infty} \psi\left(\log \frac{K(t)}{t^{\alpha}}\right)^{n} D_{J}(t) x \frac{d t}{t}
\end{aligned}
$$

by Remark 3.2 and a similar argument as in Theorem 3.5. 
In the following examples, we consider some Banach couples of function spaces, with weights $w_{0}, w_{1}$ sometimes, over the completely $\sigma$-finite measurable space $(\Omega, \mu)$, and determine the corresponding quasilogarithmic operators.

Example 3.7 (On $\left(L^{p}\left(w_{0} d \mu\right), L^{p}\left(w_{1} d \mu\right)\right)$ with $\left.1 \leq p<\infty\right)$. For $f \in L^{p}\left(w_{0} d \mu\right)+$ $L^{p}\left(w_{1} d \mu\right)$, we set

$$
f_{0}(t)=f \cdot \chi_{\left\{w_{0} \leq t^{p} w_{1}\right\}}, \quad f_{1}(t)=f \cdot \chi_{\left\{w_{0}>t^{p} w_{1}\right\}} \cdot
$$

Then $f=f_{0}(t)+f_{1}(t)$ and $D_{K}(t) f=f_{0}(t)$ by [10, Section 4.1]. It implies that

$$
\begin{aligned}
\Omega_{\psi, n}^{K}(x)=\frac{1}{n !}( & \left.-\int_{0}^{1} f_{0}(t) d \psi(\log t)^{n}+\int_{1}^{\infty} f_{1}(t) d \psi(\log t)^{n}+\psi(0)^{n} f\right) \\
=\frac{f}{n !}( & -\int_{0}^{1} \chi_{\left\{w_{0} \leq t^{p} w_{1}\right\}} d \psi(\log t)^{n} \\
& \left.+\int_{1}^{\infty} \chi_{\left\{w_{0}>t^{p} w_{1}\right\}} d \psi(\log t)^{n}+\psi(0)^{n}\right) .
\end{aligned}
$$

By dealing with two different cases, $w_{0} \leq w_{1}$ and $w_{0}>w_{1}$, we obtain that

$$
\Omega_{\psi, n}^{K}(f)=\frac{1}{n !} f \cdot \psi\left(\frac{1}{p} \log \left(\frac{w_{0}}{w_{1}}\right)\right)^{n} .
$$

For the operator $\Omega_{\psi, n}^{E_{\alpha}}$, by Remark 3.6 and a similar calculation, we obtain

$$
\Omega_{\psi, n}^{E_{\alpha}}(f)=\frac{1}{n !} f \cdot \psi\left(\frac{1}{p} \log \frac{K\left(\left(w_{0} / w_{1}\right)^{1 / p}\right)}{\left(w_{0} / w_{1}\right)^{\alpha / p}}\right)^{n} .
$$

Example 3.8 (on $\left(L^{p_{0}}\left(w_{0} d \mu\right), L^{p_{1}}\left(w_{1} d \mu\right)\right)$ with $\left.1 \leq p_{0}<p_{1} \leq \infty\right)$. For $f \in L^{p_{0}}\left(w_{0} d \mu\right)$ $+L^{p_{1}}\left(w_{1} d \mu\right)$, we set

$$
f_{0}(t)=f \cdot \chi_{\left\{|f|\left(w_{1} / w_{0}\right)^{1 /\left(p_{1}-p_{0}\right)}>t\right\}}, \quad f_{1}(t)=f \cdot \chi_{\left\{|f|\left(w_{1} / w_{0}\right)^{1 /\left(p_{1}-p_{0}\right)} \leq t\right\}} \cdot
$$

Then $f=f_{0}(t)+f_{1}(t)$ and $D_{E_{\alpha}}(t) f=f_{0}(t)$ by [10, Section 4.2]. Let $\alpha=p_{1} /\left(p_{1}-\right.$ $\left.p_{0}\right)$. A direct computation gives that

$$
\Omega_{\psi, n}^{E_{\alpha}}(f)=\frac{1}{n !} f \cdot \psi\left(\log \left(|f|\left(\frac{w_{0}}{w_{1}}\right)^{1 /\left(p_{1}-p_{0}\right)}\right)\right)^{n} .
$$

Example 3.9 (On $\left(L^{p_{0}}, L^{p_{1}}\right)$, where $L^{p_{j}}=L^{p_{j}}(\Omega, d \mu)$ with $\left.1 \leq p_{0}<p_{1} \leq \infty\right)$. For $f \in L^{p_{0}}+L^{p_{1}}$, let $r_{f}, \lambda_{f}$, and $f^{*}$ be given as before. It is known that $\lambda_{f}$ and $f^{*}$ are right continuous and nonincreasing with $f^{*}(t)=\lambda_{f}^{-1}(t)$ for each $t$ at which $f^{*}$ is continuous. Moreover, $\lim _{s \rightarrow 0} \lambda_{f}(s)=0$ and $r_{f}(\omega)=\lambda_{f}(|f(\omega)|)$. Let $1 / q=$ $1 / p_{0}-1 / p_{1}$, and let

$$
f_{0}=f \cdot \chi_{\left\{|f|>f^{*}\left(t^{q}\right)\right\}}, \quad f_{1}(t)=f \cdot \chi_{\left\{|f| \leq f^{*}\left(t^{q}\right)\right\}} .
$$


Then $f=f_{0}(t)+f_{1}(t)$ and $D_{K}(t) f=f_{0}(t)$ by [10, Section 4.3]. We can now calculate

$$
\begin{aligned}
\Omega_{\psi, n}^{K}(f)=\frac{1}{n !}( & \left.-\int_{0}^{1} f_{0}(t) d \psi(\log t)^{n}+\int_{1}^{\infty} f_{1}(t) d \psi(\log t)^{n}+\psi(0)^{n} f\right) \\
=\frac{f}{n !}( & -\int_{0}^{1} \chi_{\left\{|f|>f^{*}\left(t^{q}\right)\right\}}(t) d \psi(\log t)^{n} \\
& \left.+\int_{1}^{\infty} \chi_{\left\{|f| \leq f^{*}\left(t^{q}\right)\right\}}(t) d \psi(\log t)^{n}+\psi(0)^{n}\right) .
\end{aligned}
$$

By dealing with two different cases,

$$
r_{f}(\omega)=\left(f^{*}\right)^{-1}(|f(\omega)|) \leq 1, \quad r_{f}(\omega)=\left(f^{*}\right)^{-1}(|f(\omega)|)>1,
$$

we obtain that

$$
\Omega_{\psi, n}^{K}(f)=\frac{1}{n !} f \cdot \psi\left(\log r_{f}^{1 / q}\right)^{n}
$$

Remark 3.10. In Examples 3.8 and 3.9, on the couple $\left(L^{1}(d \mu), L^{\infty}(d \mu)\right)$, we have

$$
\Omega_{\psi, n}^{E_{1}}(f)=f \cdot \psi(\log |f|), \quad \Omega_{\psi, n}^{K}(f)=f \cdot \psi\left(\log r_{f}\right) .
$$

These operators were first studied by Kalton in [12, Section 3], and were used by him to determine the commutators of trace-class operators [13]. In those papers, Kalton developed a general commutator theorem for the rearrangement invariant function spaces with the nontrivial Boyd indices. It is an interesting problem how to put Kalton's work in the context of real interpolation [7, Section VIII].

\section{Cancellation and commutator estimates}

As a preparation for the commutator theorem, we include now the following cancellation result. The arguments on this topic have been used to deal with the classical real interpolation in [15] but are of a much more general nature, so we take them up once more.

Theorem 4.1. Let $\Phi$ be a quasi-power parameter for the real interpolation, let $\psi$ be a Lipschitz function satisfying (3.4), and let $y \in \Sigma \bar{X}$.

(i) If

$$
y=\frac{1}{n !} \int_{0}^{\infty} \psi(\log t)^{n} u(t) \frac{d t}{t}
$$

where

$$
\int_{0}^{\infty} \psi(\log t)^{k} u(t) \frac{d t}{t}=0 \quad \text { for } k=0,1, \ldots, n-1
$$


and $\|J(t, u(t))\|_{\Phi}<\infty$, then $y \in J_{\Phi}(\bar{X})$ with

$$
\|y\|_{J_{\Phi}(\bar{X})} \leq \gamma_{\psi}^{n}\|S\|_{\mathscr{S}_{(\Phi)}}^{n}\|J(t, u(t))\|_{\Phi} .
$$

(ii) If

$$
y=-\frac{1}{n !} \int_{0}^{1} x_{0}(t) d \psi(\log t)^{n}+\frac{1}{n !} \int_{1}^{\infty} x_{1}(t) d \psi(\log t)^{n},
$$

where $x_{j}: \mathbb{R}^{+} \rightarrow \Delta \bar{X}(j=0,1)$, for which $x_{0}(t)+x_{1}(t)=0$, and

$$
\int_{0}^{1} x_{0}(t) d \psi(\log t)^{k}+\int_{1}^{\infty} x_{1}(t) d \psi(\log t)^{k}=0 \quad \text { for } k=1, \ldots, n-1,
$$

with \|\|$x_{0}(t)\left\|_{0}+t\right\| x_{1}(t)\left\|_{1}\right\|_{\Phi}<\infty$, then $y \in K_{\Phi}(\bar{X})$ with

$$
\|y\|_{K_{\Phi}(\bar{X})} \leq \gamma_{\psi}^{n}\|S\|_{\mathscr{B}(\Phi)}^{n}\|\| x_{0}(t)\left\|_{0}+t\right\| x_{1}(t)\left\|_{1}\right\|_{\Phi} .
$$

Proof. (i) For $n=1$, we have

$$
\begin{aligned}
y & =\int_{0}^{\infty} \psi(\log t) u(t) \frac{d t}{t}=\int_{0}^{\infty} \psi(\log t) d \int_{0}^{t} u(s) \frac{d s}{s} \\
& =-\int_{0}^{\infty}\left(\int_{0}^{t} u(s) \frac{d s}{s}\right) \psi^{\prime}(\log t) \frac{d t}{t}
\end{aligned}
$$

in terms of integrating-by-parts and Lemma 3.4. Observe that

$$
J\left(t, \int_{0}^{t} u(s) \frac{d s}{s}\right) \leq \int_{0}^{t} J(s, u(s)) \frac{d s}{s}+t \int_{t}^{\infty} J(s, u(s)) \frac{d s}{s^{2}}
$$

according to the proof of [15, Theorem 3]. Therefore,

$$
\begin{aligned}
J\left(t, \psi^{\prime}(\log t) \int_{0}^{t} u(s) \frac{d s}{s}\right) & \leq \gamma_{\psi} J\left(t, \int_{0}^{t} u(s) \frac{d s}{s}\right) \\
& \leq \gamma_{\psi}\left(\int_{0}^{t} J(s, u(s)) \frac{d s}{s}+t \int_{t}^{\infty} J(s, u(s)) \frac{d s}{s^{2}}\right) .
\end{aligned}
$$

Since $\Phi$ is a quasi-power parameter for the real interpolation, we obtain that

$$
\left\|J\left(t, \psi^{\prime}(\log t) \int_{0}^{t} u(s) \frac{d s}{s}\right)\right\|_{\Phi} \leq \gamma_{\psi}\|S\|_{\mathscr{B}(\Phi)}\|J(t, u(t))\|_{\Phi}
$$

and hence $\|y\|_{J_{\Phi}} \leq \gamma_{\psi}\|S\|_{\mathscr{B}(\Phi)}\|J(t, u(t))\|_{\Phi}$.

For $n>1$, we have

$$
\begin{aligned}
y & =\frac{1}{n !} \int_{0}^{\infty} \psi(\log t)^{n} u(t) \frac{d t}{t}=\frac{1}{n !} \int_{0}^{\infty} \psi(\log t)^{n} d \int_{0}^{t} u(s) \frac{d s}{s} \\
& =-\frac{1}{(n-1) !} \int_{0}^{\infty}\left(\int_{0}^{t} u(s) \frac{d s}{s}\right) \psi(\log t)^{n-1} \psi^{\prime}(\log t) \frac{d t}{t}
\end{aligned}
$$


Let $v(t)=\psi^{\prime}(\log t)\left(\int_{0}^{t} u(s) d s / s\right)$. Then

$$
\begin{aligned}
y & =-\frac{1}{(n-1) !} \int_{0}^{\infty} v(t) \psi(\log t)^{n-1} \frac{d t}{t} \\
\int_{0}^{\infty} \psi(\log t)^{k} v(t) \frac{d t}{t} & =-\int_{0}^{\infty}\left(\int_{0}^{t} u(s) \frac{d s}{s}\right) \psi(\log t)^{k} \psi^{\prime}(\log t) \frac{d t}{t} \\
& =\frac{1}{k+1} \int_{0}^{\infty} \psi(\log t)^{k+1} u(t) \frac{d t}{t}=0
\end{aligned}
$$

for $k=0,1, \ldots, n-2$. By induction, we obtain that $y \in J_{\Phi}(\bar{X})$ with

$$
\|y\|_{J_{\Phi}(\bar{X})} \leq \gamma_{\psi}^{n-1}\|S\|_{\mathscr{B}(\Phi)}^{n-1}\|J(t, v(t))\|_{\Phi}
$$

By (4.10), $\|y\|_{J_{\Phi}(\bar{X})} \leq \gamma_{\psi}^{n}\|S\|_{\mathscr{B}_{(\Phi)}}^{n}\|J(t, u(t))\|_{\Phi}$.

(ii) Let $v(t)=\psi^{\prime}(\log t) x_{0}(t)$. For $n=1$, we have

$$
\begin{aligned}
y & =-\int_{0}^{1} x_{0}(t) d \psi(\log t)+\int_{1}^{\infty} x_{1}(t) d \psi(\log t) \\
& =-\int_{0}^{\infty} \psi^{\prime}(\log t) x_{0}(t) \frac{d t}{t}=-\int_{0}^{\infty} v(t) \frac{d t}{t}
\end{aligned}
$$

by assumption. Observe that

$$
\|K(t, y)\|_{\Phi} \leq\|S\|_{\mathscr{B}(\Phi)}\|J(t, v(t))\|_{\Phi}
$$

by (2.7), and

$$
\|J(t, v(t))\|_{\Phi} \leq \gamma_{\psi}\left\|J\left(t, x_{0}(t)\right)\right\|_{\Phi} \leq \gamma_{\psi}\|\| x_{0}(t)\left\|_{0}+t\right\| x_{1}(t)\left\|_{1}\right\|_{\Phi} .
$$

This gives that

$$
\|y\|_{K_{\Phi}} \leq \gamma_{\psi}\|S\|_{\Re(\Phi)}\|\| x_{0}(t)\left\|_{0}+t\right\| x_{1}(t)\left\|_{1}\right\|_{\Phi}
$$

For $n>1$, we have

$$
\int_{0}^{\infty} \psi(\log t)^{k-1} v(t) \frac{d t}{t}=\frac{1}{k} \int_{0}^{\infty} x_{0}(t) d \psi(\log t)^{k}=0
$$

for $k=1, \ldots, n-1$, and hence

$$
\begin{aligned}
y & =-\frac{1}{(n-1) !} \int_{0}^{\infty} \psi(\log t)^{n-1} v(t) \frac{d t}{t} \\
& =-\frac{1}{(n-1) !} \int_{0}^{\infty} \psi(\log t)^{n-1} d \int_{0}^{t} v(s) \frac{d s}{s} \\
& =\frac{1}{(n-1) !} \int_{0}^{\infty}\left(\int_{0}^{t} v(s) \frac{d s}{s}\right) d \psi(\log t)^{n-1} .
\end{aligned}
$$


By induction, and in view of (2.7), (4.15), and (4.16), we obtain that

$$
\begin{aligned}
\|y\|_{K_{\Phi}} & =\|K(t, y)\|_{\Phi} \leq \gamma_{\psi}^{n-1}\|S\|_{\mathscr{B}(\Phi)}^{n-1}\|J(t, v(t))\|_{\Phi} \\
& \leq \gamma_{\psi}^{n}\|S\|_{\mathscr{B}(\Phi)}^{n}\|\| x_{0}(t)\left\|_{0}+t\right\| x_{1}(t)\left\|_{1}\right\|_{\Phi},
\end{aligned}
$$

which completes the proof.

Now define the nonlinear commutators in our situation and establish the basic theorem concerning the higher order commutator estimates.

Definition 4.2. Let $T \in \mathscr{B}(\bar{X}, \bar{Y})$, let $\Omega_{n}$ be an operator from $\Sigma \bar{X}$ to $\Sigma \bar{Y}$, and let $\left[T, \Omega_{n}\right]=T \Omega_{n}-\Omega_{n} T$. The commutator $C_{n}=C_{n}(T)$ of order $n$ from $\Sigma \bar{X}$ to $\Sigma \bar{Y}$ is defined in the following way: $C_{0}(T)=T, C_{1}(T)=\left[T, \Omega_{1}\right]$, and

$$
C_{n}(T)=\left[T, \Omega_{n}\right]-\sum_{k=1}^{n-1} \Omega_{k} C_{n-k} \quad \text { for } n \geq 2 .
$$

In particular, for $\Omega_{n}=\Omega_{\psi, n}^{J}, \Omega_{\psi, n}^{K}, \Omega_{\psi, n}^{E}$ or $\Omega_{\psi, n}^{\alpha}$, we write $C_{n}=C_{\psi, n}^{J}, C_{\psi, n}^{K}, C_{\psi, n}^{E}$ or $C_{\psi, n}^{\alpha}$.

Theorem 4.3. Assume that $\Phi$ is a quasi-power parameter for the real interpolation, and assume that $\psi$ is a Lipschitz function satisfying (3.4). Then, in each of the following cases, there exists a constant $A$ depending on $n, \Phi$, and $\psi$ such that

$$
\begin{gathered}
\left\|C_{\psi, n}^{J}(T) x\right\|_{J_{\Phi}(\bar{Y})} \leq A\|T\|_{J_{\Phi}(\bar{X}), J_{\Phi}(\bar{Y})}\|x\|_{J_{\Phi}(\bar{X})}, \\
\left\|C_{\psi, n}^{K}(T) x\right\|_{K_{\Phi}(\bar{Y})} \leq A\|T\|_{K_{\Phi}(\bar{X}), K_{\Phi}(\bar{Y})}\|x\|_{K_{\Phi}(\bar{X})}, \\
\left\|C_{\psi, n}^{E}(T) x\right\|_{E_{\Phi}(\bar{Y})} \leq A\|T\|_{E_{\Phi}(\bar{X}), E_{\Phi}(\bar{Y})}\|x\|_{E_{\Phi}(\bar{X})},
\end{gathered}
$$

for all $x \in J_{\Phi}(\bar{X}), K_{\Phi}(\bar{X})$, or $E_{\Phi}(\bar{X})$, respectively. If $0<\theta<1$ and $1 \leq p<\infty$, then there exists a constant $A$ depending on $\alpha, \psi, n, \theta$, and $p$ such that

$$
\left\|C_{\psi, n}^{\alpha}(T) x\right\|_{\bar{Y}_{\theta, p, E_{\alpha}}} \leq A\|T\|_{\bar{X}_{\theta, p, E_{\alpha}}, \bar{Y}_{\theta, p, E_{\alpha}}}\|x\|_{\bar{X}_{\theta, p, E_{\alpha}}}
$$

for all $x \in \bar{X}_{\theta, p, E_{\alpha}}$.

Proof. We mention first that all estimates are trivial for $n=0$ since $C_{0}=T$.

For (4.22), we have $C_{1}(T)=\left[T, \Omega_{1}\right]$ and hence

$$
C_{1}(T) x=\int_{0}^{\infty}\left(T\left(D_{J}(t) x\right)-D_{J}(t)(T x)\right) \psi(\log t) \frac{d t}{t}
$$

for all $x \in J_{\Phi}(\bar{X})$. Let

$$
u_{1}(t)=T\left(D_{J}(t) x\right)-D_{J}(t)(T x)
$$


254 Commutators in real interpolation

Then $\int_{0}^{\infty} u_{1}(t) d t / t=0$. According to Theorem 4.1(i), we have

$$
\begin{aligned}
\left\|C_{1}(T) x\right\|_{J_{\Phi}(\bar{Y})} & \leq \gamma_{\psi}\|S\|_{\mathscr{B}(\Phi)}\left\|J\left(t, u_{1}(t) ; \bar{Y}\right)\right\|_{\Phi} \\
& \leq \gamma_{\psi}\|S\|_{\mathscr{B}(\Phi)}\left(\left\|J\left(t, T\left(D_{J}(t) x\right) ; \bar{Y}\right)\right\|_{\Phi}+\left\|J\left(t, D_{J}(t)(T x) ; \bar{Y}\right)\right\|_{\Phi}\right) \\
& \leq 2 c \gamma_{\psi}\|S\|_{\mathscr{B}(\Phi)}\|T\|_{J_{\Phi}(\bar{X}), J_{\Phi}(\bar{Y})}\|x\|_{J_{\Phi}(\bar{X})} .
\end{aligned}
$$

Furthermore, we have

$$
C_{2}(T) x=\frac{1}{2} \int_{0}^{\infty} \psi(\log t)^{2} u_{1}(t) \frac{d t}{t}-\int_{0}^{\infty} \psi(\log t) u_{2}(t) \frac{d t}{t}
$$

for all $x \in J_{\Phi}(\bar{X})$, where

$$
\int_{0}^{\infty} u_{2}(t) \frac{d t}{t}=\int_{0}^{\infty} \psi(\log t) u_{1}(t) \frac{d t}{t}=C_{1}(T) x .
$$

This, together with Lemma 3.4, implies that

$$
C_{2}(T) x=\int_{0}^{\infty}\left(-\psi^{\prime}(\log t) \int_{0}^{t} u_{1}(s) \frac{d s}{s}-u_{2}(t)\right) \psi(\log t) \frac{d t}{t},
$$

meanwhile

$$
\begin{aligned}
\int_{0}^{\infty} & \left(-\psi^{\prime}(\log t) \int_{0}^{t} u_{1}(s) \frac{d s}{s}-u_{2}(t)\right) \frac{d t}{t} \\
& =\int_{0}^{\infty} \psi(\log t) u_{1}(t) \frac{d t}{t}-\int_{0}^{\infty} u_{2}(t) \frac{d t}{t}=0
\end{aligned}
$$

By Theorem 4.1(i) again, we obtain

$$
\begin{aligned}
& \left\|C_{2}(T) x\right\|_{J_{\Phi}(\bar{Y})} \leq \gamma_{\psi}\|S\|_{\mathscr{B}(\Phi)}\left(\left\|J\left(t, \psi^{\prime}(\log t) \int_{0}^{t} u_{1}(s) \frac{d s}{s} ; \bar{Y}\right)\right\|_{\Phi}\right. \\
& \left.+\left\|J\left(t, u_{2}(t) ; \bar{Y}\right)\right\|_{\Phi}\right)
\end{aligned}
$$

whereas

$$
\begin{gathered}
\left\|J\left(t, \psi^{\prime}(\log t) \int_{0}^{t} u_{1}(s) \frac{d s}{s} ; \bar{Y}\right)\right\|_{\Phi} \leq 2 c \gamma_{\psi}\|S\|_{\mathscr{B}(\Phi)}\|T\|_{J_{\Phi}(\bar{X}), J_{\Phi}(\bar{Y})}\|x\|_{J_{\Phi}(\bar{X})}, \\
\left\|J\left(t, u_{2}(t) ; \bar{Y}\right)\right\|_{\Phi} \leq 2 c \gamma_{\psi}\|S\|_{\mathscr{B}(\Phi)}\|T\|_{J_{\Phi}(\bar{X}), J_{\Phi}(\bar{Y})}\|x\|_{J_{\Phi}(\bar{X})} .
\end{gathered}
$$

Therefore, we can prove (4.22) inductively for all $n \geq 2$. 
For (4.23), we set $x_{0}(t)=D_{K}(t) x$ and $x_{1}(t)=x-x_{0}(t)$ for all $x \in K_{\Phi}(\bar{X})$. Thus

$$
\begin{aligned}
C_{1}(T) x= & -\int_{0}^{1}\left(T x_{0}(t)-(T x)_{0}(t)\right) d \psi(\log t) \\
& +\int_{1}^{\infty}\left(T x_{1}(t)-(T x)_{1}(t)\right) d \psi(\log t) .
\end{aligned}
$$

Let $y_{0}(t)=T x_{0}(t)-(T x)_{0}(t)$ and $y_{1}(t)=T x_{1}(t)-(T x)_{1}(t)$. Then

$$
y_{0}(t)+y_{1}(t)=T x-T x=0 .
$$

According to Theorem 4.1(ii), we have

$$
\begin{aligned}
&\left\|C_{1}(T) x\right\|_{K_{\Phi}(\bar{Y})} \leq \gamma_{\psi}\|S\|_{\Re(\Phi)} \|\left\|y_{0}(t)\right\|_{0}+t\left\|y_{1}(t)\right\|_{1} \|_{\Phi} \\
& \leq \gamma_{\psi}\|S\|_{\Re(\Phi)}(\|\| T x_{0}(t)\left\|_{0}+t\right\| T x_{1}(t)\left\|_{1}\right\|_{\Phi} \\
&\left.+\|\|(T x)_{0}(t)\left\|_{0}+t\right\|(T x)_{1}(t)\left\|_{1}\right\|_{\Phi}\right) \\
& \leq 2 c \gamma_{\psi}\|S\|_{\Re(\Phi)}\|T\|_{K_{\Phi}(\bar{X}), K_{\Phi}(\bar{Y})}\|x\|_{K_{\Phi}(\bar{X})} .
\end{aligned}
$$

The case $n>1$ and the estimates in (4.24) and (4.25) can be proved by making a simple substitution or by induction as we did above. We leave all details to the reader.

\section{Final remarks}

Remark 5.1. According to Theorem 4.3, $C_{n}$ can be considered as a bounded (nonlinear) operator on $\mathscr{B}(\bar{X}, \bar{Y})$. This uniform boundedness plays an important role in future studies.

Remark 5.2. According to Theorem 4.3, operators $\Omega_{\psi, 1}^{J}$ and $\Omega_{\psi, 1}^{K}$ are uniquely defined up to bounded errors. Combine this fact with Theorem 3.5, we may simply write $\Omega_{\psi}=\Omega_{\psi, 1}^{J}=\Omega_{\psi, 1}^{K}$. Observe that if $\psi$ is a bounded function, then

$$
\Omega_{\psi}=\int_{0}^{\infty} \psi(\log t) D_{J}(t) x \frac{d t}{t}
$$

is a bounded operator on $J_{\Phi}(\bar{X})=K_{\Phi}(\bar{X})$ and vice versa.

Remark 5.3. If we replace the natural number $n$ by any positive real number $\eta$ in Definitions 3.1 and 4.2, then we can obtain the quasilogarithmic operators $\Omega_{\psi, \eta}^{J}$ and $\Omega_{\psi, \eta}^{K}$, and the corresponding commutators $C_{\psi, \eta}^{J}$ and $C_{\psi, \eta}^{K}$, of fractional order $\eta$. There are no essential difficulties to carry over Theorems 3.5, 4.1, and 4.3 to this more general case. In particular, if one chooses $\Phi=L_{\varphi}^{p}$ as in Example 2.2 and $\psi(t)=t,[1$, Theorem 8.5$]$ is recovered. 
Remark 5.4. Let $(\Omega, \mu)$ be a completely $\sigma$-finite measurable space, and let $X$ be an r.i. function space over $(\Omega, \mu)$ with the nontrivial Boyd indices $\alpha$ and $\beta$, that is, $0<\alpha \leq \beta<1$. One can construct a quasi-power parameter $\Phi$ for the real interpolation for which $X=K_{\Phi}\left(L^{1}, L^{\infty}\right)=J_{\Phi}\left(L^{1}, L^{\infty}\right)$, where $L^{p}=L^{p}(\Omega, d \mu)$ for $1 \leq p \leq \infty$. In fact, by the Luxemberg representation theorem [2, Theorem II.4.10], there is an r.i. function space $\hat{X}$ over $\left(\mathbb{R}^{+}, d t\right)$ such that $\|f\|_{X}=\left\|f^{*}\right\|_{\hat{X}}$ for all $f \in X$. Now define the function space $\Phi$ over $\left(\mathbb{R}^{+}, d t / t\right)$ by $\Phi=\{f \mid f(t) / t \in$ $\hat{X}\}$ with the norm $\|f\|_{\Phi}=\|f(t) / t\|_{\hat{X}}$. If we choose $p_{0}$ and $p_{1}$ with $1<p_{0}<$ $1 / \beta \leq 1 / \alpha<p_{1}<\infty$, then, according to the Boyd interpolation theorem [2, Theorem III.5.16], $\hat{X}$ and its Köthe dual $(\hat{X})^{\prime}$ are interpolation spaces for the couple $\left(L^{p_{0}}, L^{p_{1}}\right)$. As a consequence, the Calderón operator $S$ is bounded on $\Phi$. In addition, $1 \wedge(1 / t) \in L^{p_{0}} \cap L^{p_{1}} \subseteq \hat{X} \cap(\hat{X})^{\prime}$. This implies that $1 \wedge t \in \Phi$ and

$$
\int_{0}^{\infty} 1 \wedge\left(\frac{1}{t}\right)|f(t)| \frac{d t}{t} \leq\left\|1 \wedge\left(\frac{1}{t}\right)\right\|_{(\hat{X})^{\prime}} \cdot\left\|\frac{f(t)}{t}\right\|_{\hat{X}}<\infty
$$

for all $f \in \Phi$. Therefore, $\Phi$ is a quasi-power parameter for the real interpolation. The equivalence $X=K_{\Phi}\left(L^{1}, L^{\infty}\right)$ can be proved as in [8, Section 1]. With this construction, we may apply Theorem 4.3 on the r.i. space $X$ with the nontrivial Boyd indices, and obtain the commutator theorem for operators $\Omega_{\psi, 1}^{K}$, which are given in Remark 3.10, on $X$. This is the case considered by Kalton [12, Corollary 3.2]. By applying Theorem 4.3 on the noncommutative $L^{p}$-spaces, the Schatten classes $C_{p}$, one can immediately get other results by Kalton [13, Theorems 4.2 and 4.3]. The methods used by Kalton to prove those results are totally different.

\section{References}

[1] J. Bastero, M. Milman, and F. J. Ruiz, On the connection between weighted norm inequalities, commutators and real interpolation, Mem. Amer. Math. Soc. 154 (2001), no. 731, viii+80.

[2] C. Bennett and R. Sharpley, Interpolation of Operators, Pure and Applied Mathematics, vol. 129, Academic Press, Massachusetts, 1988.

[3] J. Bergh and J. Löfström, Interpolation Spaces. An Introduction, Grundlehren Math. Wiss., vol. 223, Springer-Verlag, Berlin, 1976.

[4] Yu. A. Brudnyı̆ and N. Ya. Krugljak, Interpolation Functors and Interpolation Spaces. Vol. I, North-Holland Publishing, Amsterdam, 1991.

[5] M. J. Carro, J. Cerdà, and J. Soria, Higher order commutators in interpolation theory, Math. Scand. 77 (1995), no. 2, 301-319.

[6] M. Cwikel, B. Jawerth, and M. Milman, The domain spaces of quasilogarithmic operators, Trans. Amer. Math. Soc. 317 (1990), no. 2, 599-609.

[7] M. Cwikel, B. Jawerth, M. Milman, and R. Rochberg, Differential estimates and commutators in interpolation theory, Analysis at Urbana, Vol. II (Urbana, IL, 19861987), Cambridge University Press, Cambridge, 1989, pp. 170-220.

[8] M. Cwikel and E. Pustylnik, Weak type interpolation near "endpoint" spaces, J. Funct. Anal. 171 (2000), no. 2, 235-277.

[9] T. Holmstedt, Interpolation of quasi-normed spaces, Math. Scand. 26 (1970), 177199. 
[10] B. Jawerth, R. Rochberg, and G. Weiss, Commutator and other second order estimates in real interpolation theory, Ark. Mat. 24 (1986), no. 2, 191-219.

[11] S. Kaijser, A new proof of the fundamental lemma of interpolation theory, Math. Scand. 78 (1996), no. 2, 266-270.

[12] N. J. Kalton, Nonlinear commutators in interpolation theory, Mem. Amer. Math. Soc. 73 (1988), no. 385, iv+85.

[13] _ , Trace-class operators and commutators, J. Funct. Anal. 86 (1989), no. 1, 4174 .

[14] M. Milman, Higher order commutators in the real method of interpolation, J. Anal. Math. 66 (1995), 37-55.

[15] M. Milman and R. Rochberg, The role of cancellation in interpolation theory, Harmonic Analysis and Operator Theory (Caracas, 1994), American Mathematical Society, Rhode Island, 1995, pp. 403-419.

[16] R. Rochberg, Higher order estimates in complex interpolation theory, Pacific J. Math. 174 (1996), no. 1, 247-267.

[17] R. Rochberg and G. Weiss, Derivatives of analytic families of Banach spaces, Ann. of Math. (2) 118 (1983), no. 2, 315-347.

Ming Fan: Institute of Mathematics and Natural Sciences, Dalarna UniverSity College, 78188 Borlänge, Sweden

E-mail address: fmi@du.se 


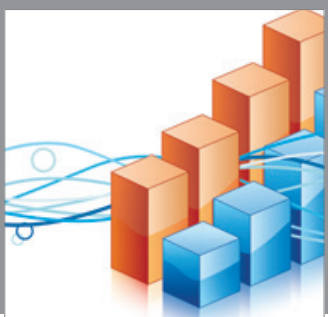

Advances in

Operations Research

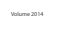

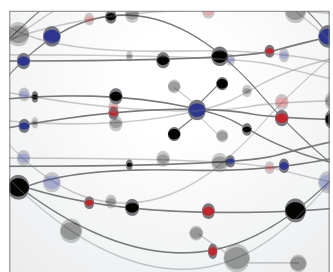

\section{The Scientific} World Journal
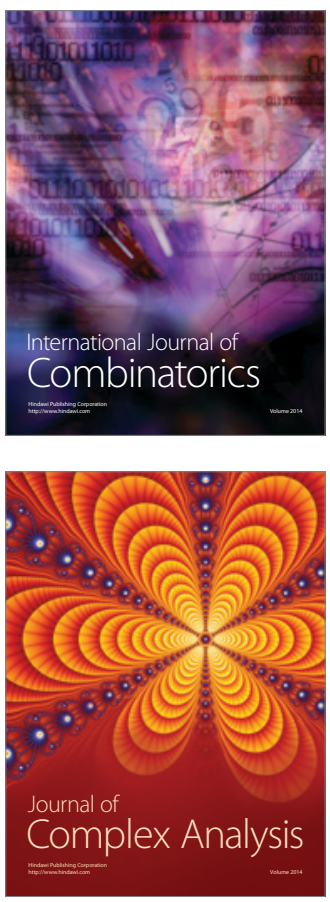

International Journal of

Mathematics and

Mathematical

Sciences
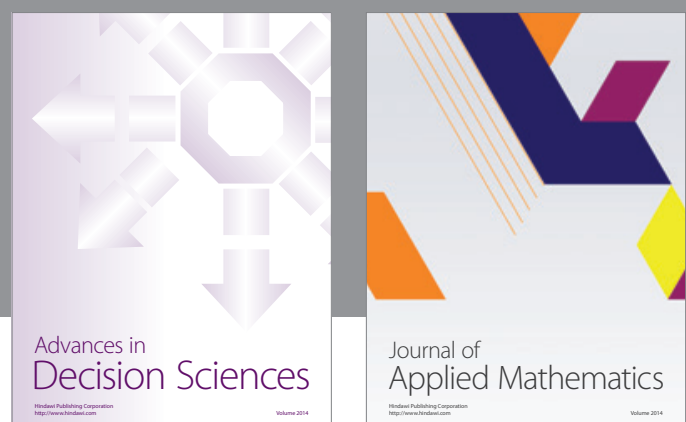

Journal of

Applied Mathematics
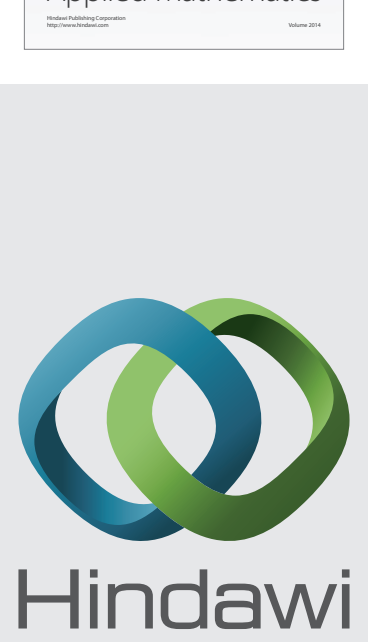

Submit your manuscripts at http://www.hindawi.com
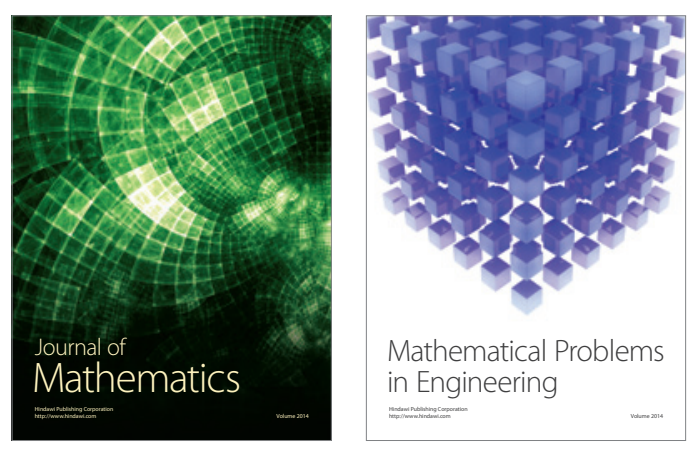

Mathematical Problems in Engineering
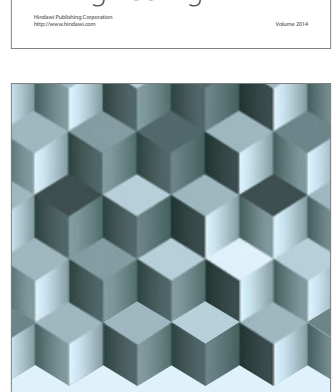

Journal of

Function Spaces
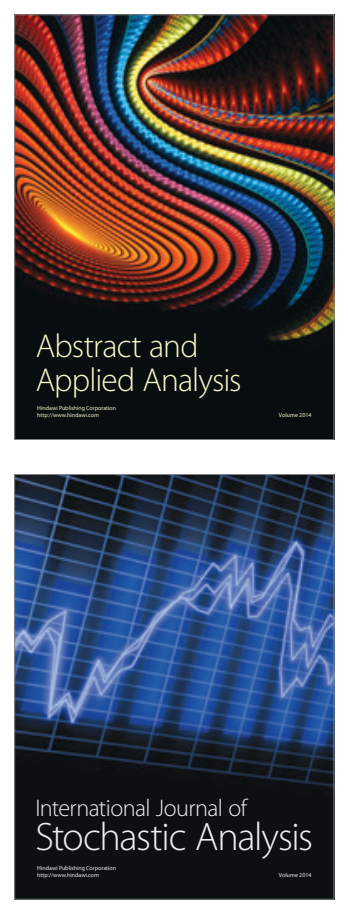

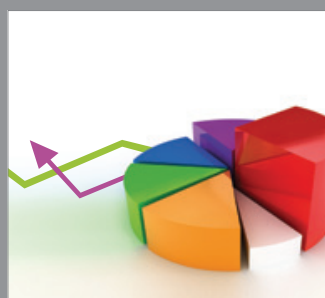

ournal of

Probability and Statistics

Promensencen
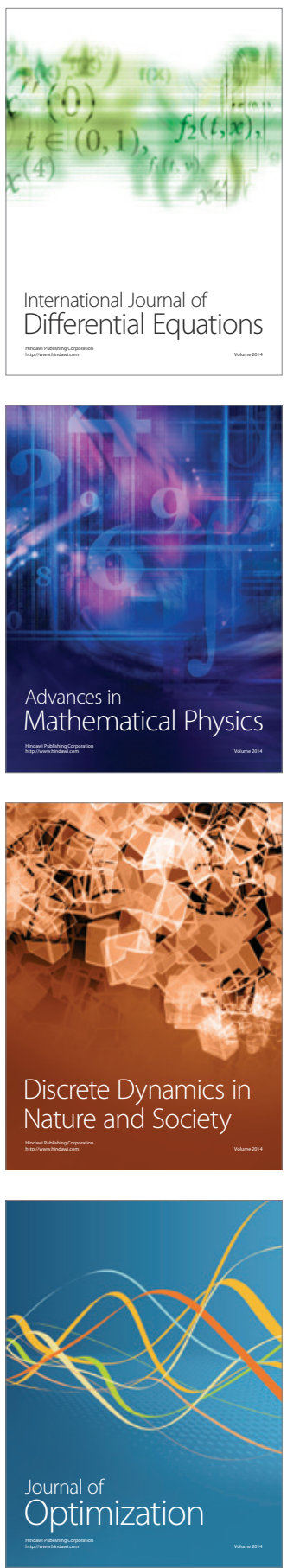